\title{
Multislice computed tomography and X-ray correlation of the classical secondary vascular adaptive features associated with aortic coarctation
}

\author{
Elliott M. Groves ${ }^{1,2}$, Pranav M. Patel ${ }^{1}$ \\ 1. Division of Cardiology, Department of Medicine, University of California, Irvine, CA, USA. 2. Department of Biomedical \\ Engineering, University of California, Irvine, CA, USA.
}

Correspondence: Elliott M. Groves. Address: Division of Cardiology, Department of Medicine, UC Irvine School of Medicine, 333 City Blvd. West, Suite 400 Orange, CA 92868-3298, USA. Email: egroves@uci.edu

Received: December 23, 2014

DOI : $10.5430 /$ ijdi.v2n2p60
Accepted: April 13, 2015

URL: http://dx.doi.org/10.5430/ijdi.v2n2p60

\section{Abstract}

Coarctation of the aorta is a discrete narrowing of the vessel which most commonly presents juxtaductal, distal to origin of the left subclavian artery. Its etiology is generally congenital and the resulting pressure overload can result in several adaptive vascular features. Here we present a case of a Hispanic male patient who presented with a subarachnoid hemorrhage due to malignant hypertension which was the result of a long standing undiagnosed coarctation. Due to the long standing nature of this patients congenital defect he had a striking example of many of the vascular adaptations associated with coarctation. Ultimately, a successful open surgical repair was undertaken which restored normal perfusion to the lower extremities and normalized upper extremity blood pressure.

\section{Keywords}

Coarctation, Aorta, Intercostal artery, Subarachnoid hemorrhage, Thoracic surgery, Computed tomography

\section{I ntroduction}

Coarctation of the aorta accounts for 4\%-6\% of all congenital heart defects and has a prevalence of 4 per 10,000 live births

${ }^{[1]}$. It is characterized by a discrete narrowing typically of the thoracic aorta juxtaductal, distal to the origin of the left subclavian artery ${ }^{[2]}$. The vast majority of cases are congenital and the exact pathogenesis is unknown. There are several cardiac and non-cardiac manifestations of coarctation. These include elevated blood pressure in the upper extremities, weak lower extremity pulses with brachial-femoral delay, rib notching due to robust collateral circulation, intracranial hemorrhage due to increased risk of intracranial aneurysms and Turners syndrome ${ }^{[3]}$. Diagnosis is typically made in the post-natal phase with a variety of imaging techniques. However presentation and time to diagnosis varies depending upon the age of the patient and the severity of the lesion. Timely recognition and diagnosis of this abnormality is important to plan and execute a surgical repair before the patient suffers consequences from the longstanding hypertension and the other adaptive features. 


\section{Case presentation}

A 34-year-old Hispanic gentleman with poorly controlled hypertension presented with the sudden onset of a headache, which was described as the "worst headache of my life". His initial average blood pressure in the upper extremities was 230/118 mm Hg and 99/69 mm Hg in the lower extremities (standard deviation in upper extremities 7/2.8 and 1.4/2.8 in the lower extremities). Physical exam was significant for auscultation of a systolic murmur in the left infraclavicular area and under the left scapula. Palpation of the pedal pulses revealed 2+ symmetrical upper extremity pulses, absent femoral, dorsalis pedis and posterior tibial pulses bilaterally. Further history revealed chronic complaints of cold lower extremities and occasional chest discomfort.

An initial computed axial tomography (CT) scan of the head demonstrated a subarachnoid hemorrhage (SAH). Chest X-Ray revealed bilateral rib notching, mild dilatation of the ascending aorta, localized narrowing of the distal aortic arch, and mild dilatation of the descending aorta. This silhouette of the aorta indicating prestenotic and poststenotic dilatation resulted in a "Figure 3" appearance of the aorta that was consistent with coarctation (see Figure 1). CT coronary and aortic angiography revealed severe tubular coarctation with severe hypoplasia of the isthmus in a $1 \mathrm{~cm}$ segment at the level of tracheal bifurcation and distal to the left subclavian artery. There was evidence of extensive intercostal and intramammary collaterals (see Figures 2-3). Rib notching was also evident on CT imaging. Patient was then referred to cardiothoracic surgery for open repair (see Figure 3). The repair was a success, restored normal perfusion to the lower extremities and normalized upper extremity blood pressure. Ultimately the patient was discharged with no complications and has not been readmitted to the hospital.

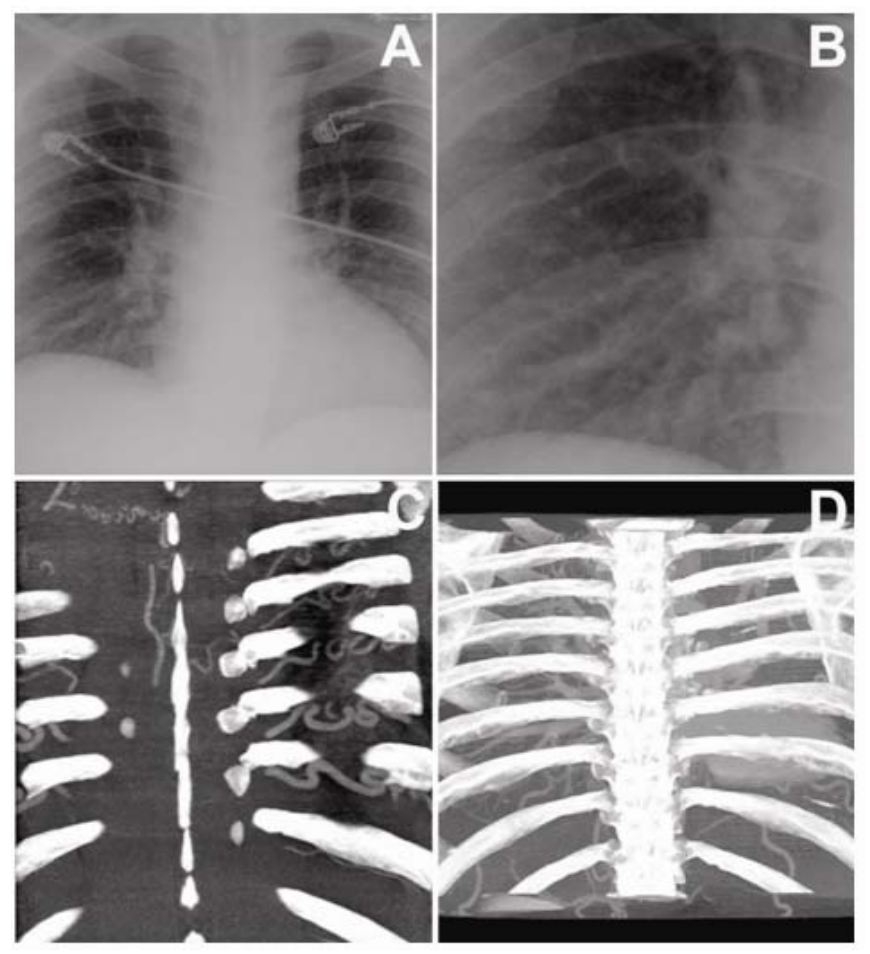

Figure 1. Four images illustrating the striking rib notching present in this patient. (A) is an Anterior-Posterior Chest Film and (B) is a magnified image of the rib notching on the right. (C) is a Chest CT Coronal image clearly showing the dramatic dilation of the intercostal arteries, which lead to rib notching as described in text. (D) is a reconstructed image of the bony structures of the thorax from the 64 slice CT Angiogram, again illustrating dramatic rib notching throughout the chest. 

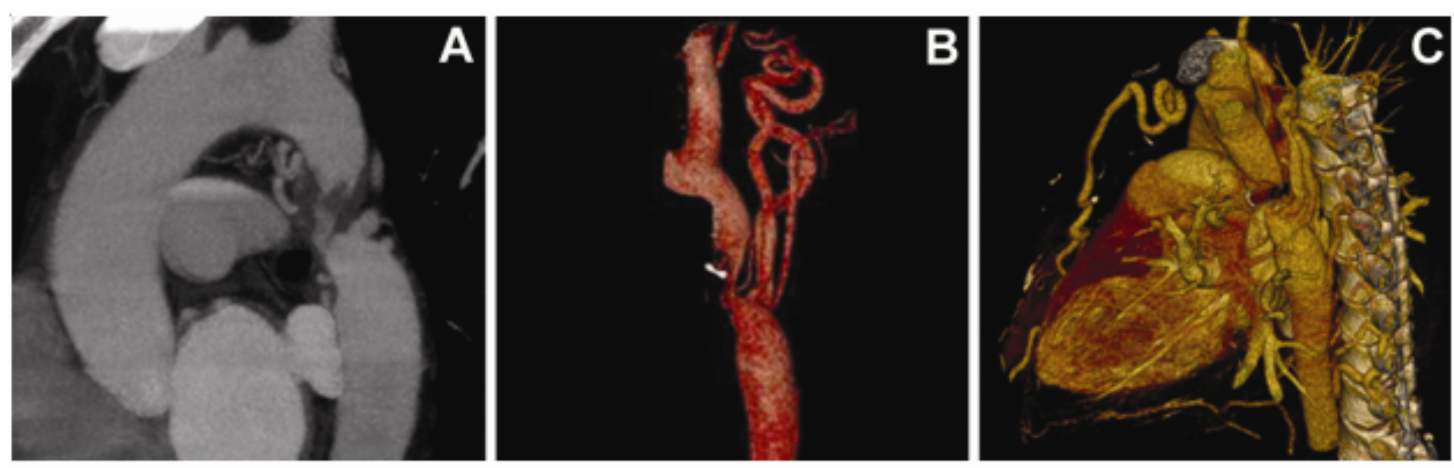

Figure 2. CT Angiography revealing a high grade coarctation. (A) is a sagittal image from a CT Angiogram of the Aorta revealing substantial narrowing of the descending thoracic aorta with a limited amount of flow distal to the coarctation. (B) is a three dimensional reconstruction of the CT Angiography which again reveals a high grade coarctation. Both images also clearly show a markedly dilated L subclavian artery. Three dimensional reconstruction of the 64 slice CT Coronary Angiogram in (C) shows extraordinarily large collaterals feeding the thoracic aorta distal to the obstruction. Additionally, the dilated internal mammary arteries are seen coursing anteriorly.
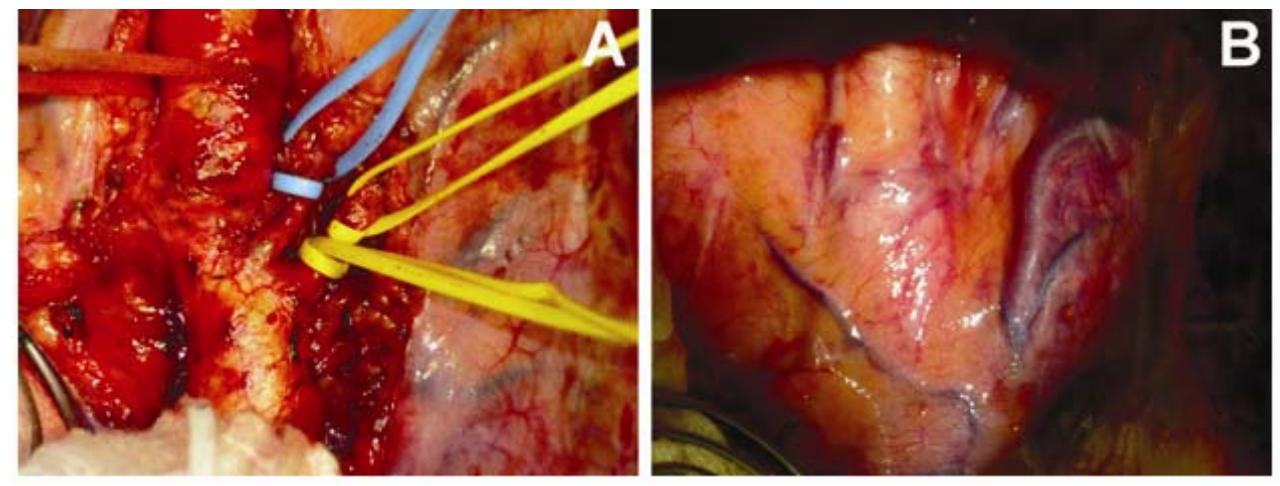

Figure 3. These are intra operative photos taken at the time of open repair. (A) shows the coarctation itself with large collateral vessels joining the aorta distal to the obstruction. (B) is an image of the strikingly dilated collateral circulation, in this case intercostal arteries.

\section{Discussion}

Coarctation of the Aorta is a discrete narrowing of the vessel which most commonly presents juxtaductal, distal to origin of the left subclavian artery ${ }^{[2]}$. There are a variety of cardiac abnormalities which present more commonly in patients with coarctation, such as bicuspid aortic valve, ventricular septal defect and patent ductus arteriosus ${ }^{[4]}$. In the setting of aortic coarctation there are secondary or noncardiac vascular abnormalities that are present especially in cases of long standing disease in the absence of any treatment. Collateral circulation to the vascular beds distal to the narrowed segment of aorta lead to markedly dilated internal mammary, intercostal, subclavian and scapular arteries ${ }^{[2]}$. As previously stated, in the setting of untreated disease the degree of collateralization can be extraordinary. This case is an excellent example of the secondary vascular abnormalities in both the degree of dilatation of the collateral circulation and the illustration by the accompanying imaging. Large intercostal arteries can be seen as can a grossly dilated left subclavian and internal mammary artery. Numerous imaging modalities can provide illustration of the coarct itself and the accompanying collateral circulation which also result in anatomic changes such as rib notching. Chest radiography, as in this case, can reveal notching of most commonly the third to eighth ribs, due to erosion by large collateral arteries, notching increases with age and is most frequently seen in the posterior ribs as the anterior intercostal arteries do not lie in costal 
grooves ${ }^{[2,5]}$. CT Angiography can provide a high resolution image for diagnosis of the degree and location of the coarctation, in addition to providing a clear display of the further collateral circulation such as the subclavian and internal mammary arteries ${ }^{[2]}$. Treatment options include surgical repair as utilized in this case, balloon angioplasty, and stent placement.

\section{References}

[1] Hoffman JI, Kaplan S. The incidence of congenital heart disease. Journal of the American College of Cardiology. 2002; 39(12): 1890-900. http://dx.doi.org/10.1016/S0735-1097(02)01886-7

[2] Brickner ME, Hillis LD, Lange RA. Congenital heart disease in adults. New England Journal of Medicine. 2000; $342(4)$ : $256-63$. PMid:10648769 http://dx.doi.org/10.1056/NEJM200001273420407

[3] Hodes H, Steinfeld L, Blumenthal S. Congenital cerebral aneurysms and coarctation of the aorta. Archives of pediatrics. 1959; 76(1): 28. PMid:13618092

[4] Nihoyannopoulos P, et al. Accuracy of two-dimensional echocardiography in the diagnosis of aortic arch obstruction. Journal of the American College of Cardiology. 1987; 10(5): 1072-77. http://dx.doi.org/10.1016/S0735-1097(87)80348-0

[5] Tobian L. A viewpoint concerning the enigma of hypertension. The American journal of medicine. 1972; 52(5): 595-609. http://dx.doi.org/10.1016/0002-9343(72)90051-4 